\section{Unique Minilens Cooling System Results in a Compact TEM}

Dmitry Lysenkov ${ }^{1}$, and William C. Monigle ${ }^{2}$ Carl Zeiss SMT AG, Nano Technology Systems Division,

${ }^{1}$ Oberkochen, Germany and ${ }^{2}$ Peabody, MA

d.lysenkov@smt.zeiss.com

\section{Introduction}

Transmission electron microscopy (TEM) has been proven to be one of the most convenient and straight forward methods for resolving sub-nanometer structures. The use of these instruments in life sciences, materials sciences and in the semiconductor industry has contributed greatly to many significant advances. However, typical TEMs are very large, expensive and relatively complicated to operate. Fortunately, recent advances in TEM technology and product design have created a new TEM instrument that is very well suited to everyday applications in hospitals, universities and laboratories of all types. Able to fit in a small room, this new TEM does not require the expensive, extensive building modifications usually associated with a TEM installation. The small size of the instrument is due to the instrument's column design, which features two condenser lenses, a symmetrical objective lens and four projective minilenses. In particular, the minilens design is largely responsible for providing the compact size of the instrument with its small footprint, plus its higher contrast imaging with less distortion.

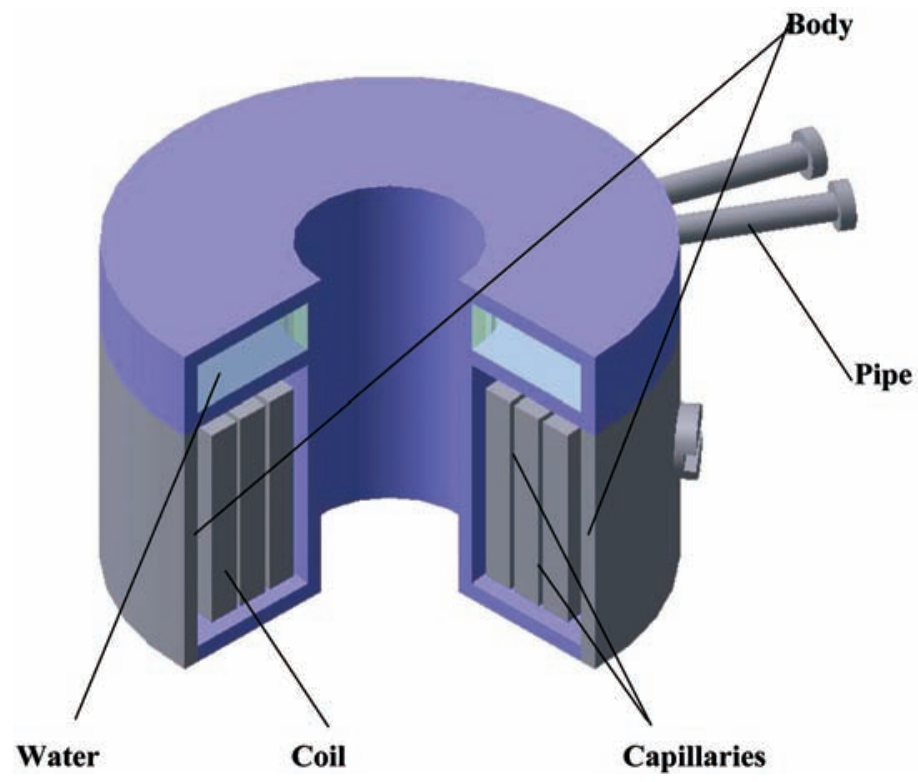

Fig. 1: Lens coil body of minilens with water cooling of upper lid

\section{Minilens Design}

This minilens design uses a unique cooling system based on principles developed by T. Mulvey [1]. This approach enables lens operation at high current densities, plus a decreased size of the lens while maintaining both thermal and current stability of the coil. Without controlling the temperature of the coil, the properties of the lenses will change, diminishing the performance of the microscope.

The lens coils are situated in a chamber filled with a liquid whose vaporization is used for cooling. (Fig. 1) As soon as the coil becomes hot, the liquid rises within the coil by capillary forces,

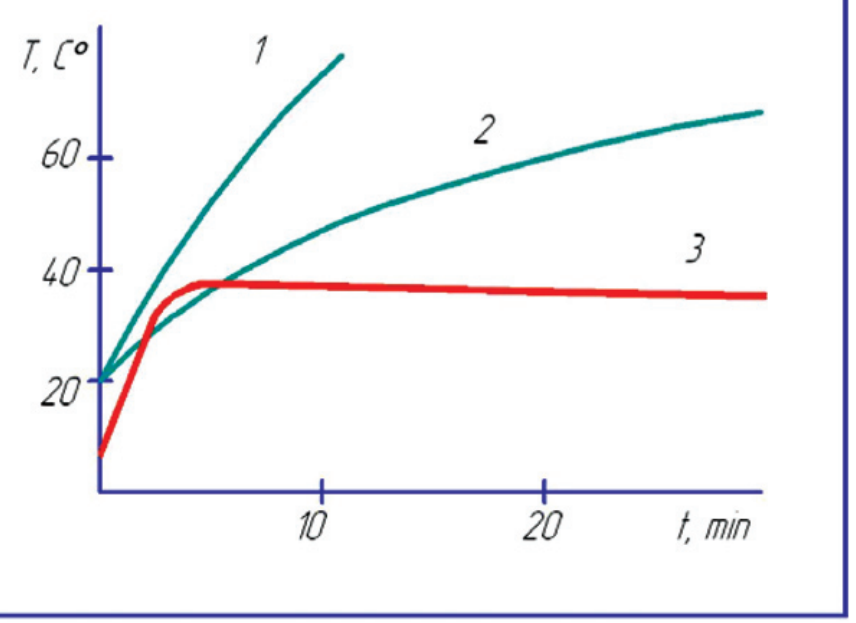

Fig. 2: Temperature profile of electromagnetic lens coils with different designs: 1 Non-cooled lens coil at a current density of $3 \mathrm{~A} / \mathrm{mm}^{2} ; 2$ Lens coil with a water cooled surface at a current density of $3 \mathrm{~A} / \mathrm{mm}^{2} ; 3 \mathrm{New}$ lens coil with vaporization cooling at a current density of $10 \mathrm{~A} / \mathrm{mm}^{2}$.

evaporating when the temperature increases. The upper lid of the coil chamber is cooled by water, so the vapor condenses when it reaches the lid there and flows down back into the coil chamber. In accordance with Mulvey's principles, it is sufficient to have the coils only partially immersed in the liquid.

This "heat pipe" approach is clearly more efficient than conventional techniques used for cooling lenses. After switch-on of the system, the temperature of the coil rises quickly up to the vaporization point of the liquid and remains stable at this point despite changes in the current density in the coils, which is necessary for changes in magnification and illumination conditions of

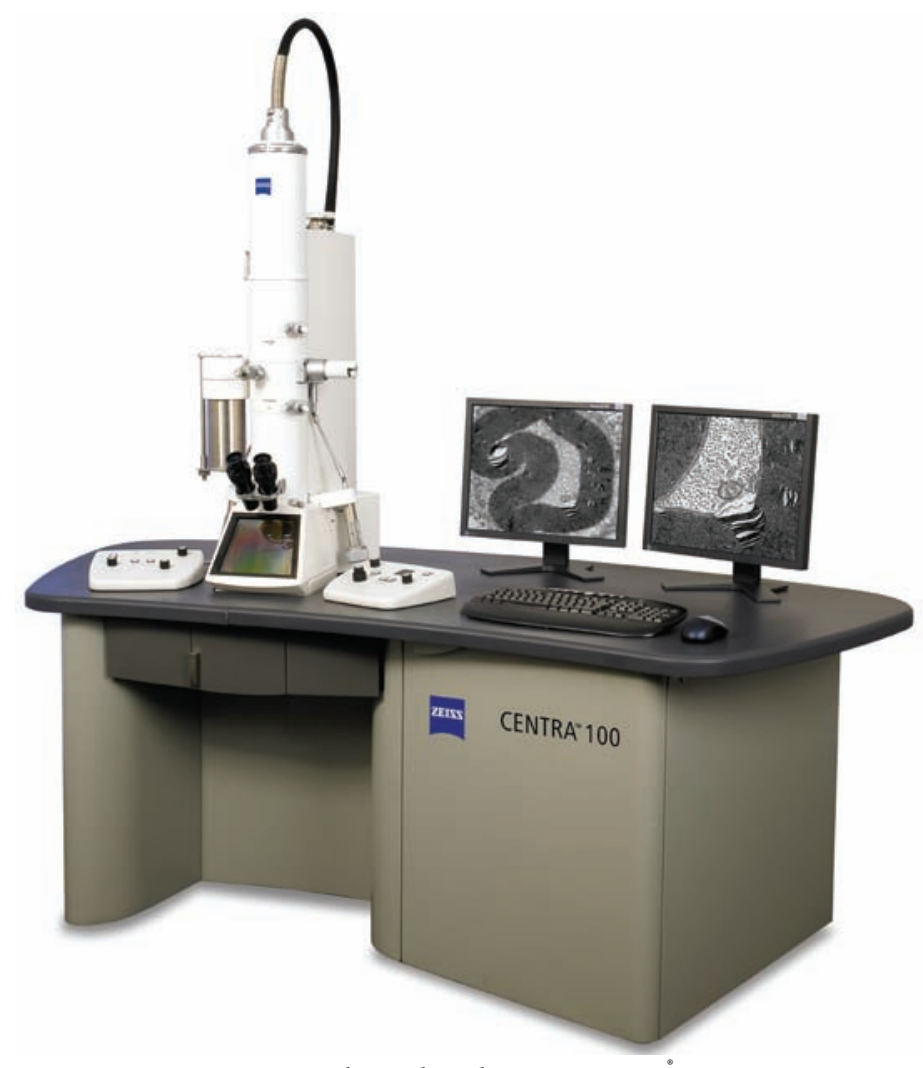

Fig. 3: New TEM with minilens design: CENTRA 100 


\section{POWER THROUEH SIMPLICITY - AN IMAEING BOLUTION YOU COULD ONLY DAEAM ABOUT}

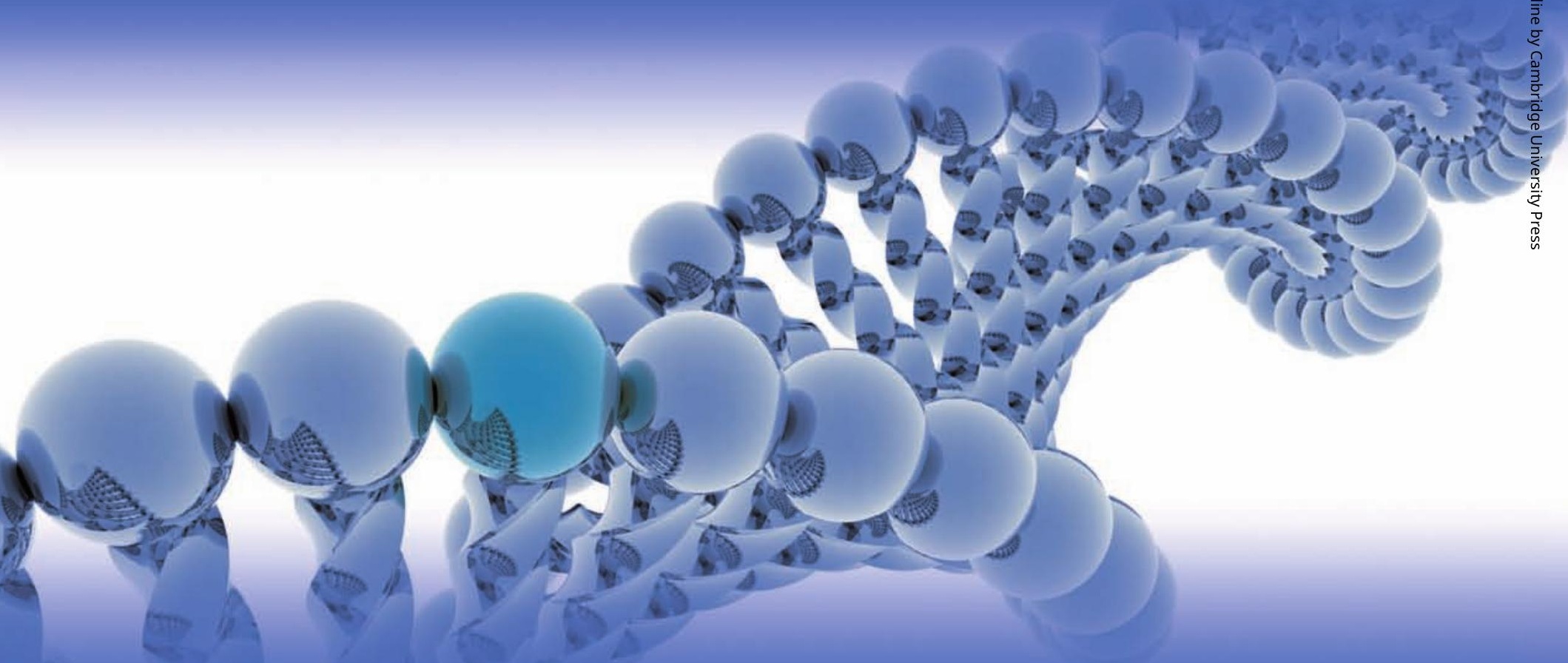

\section{ZEISS CENTRA® 100}

An efficient, fully digital, and robust

multi-purpose 100 kV TEM.

Designed for rapid biomedical applications.

CENTRA $^{\circledR} 100$ delivers outstanding image quality.
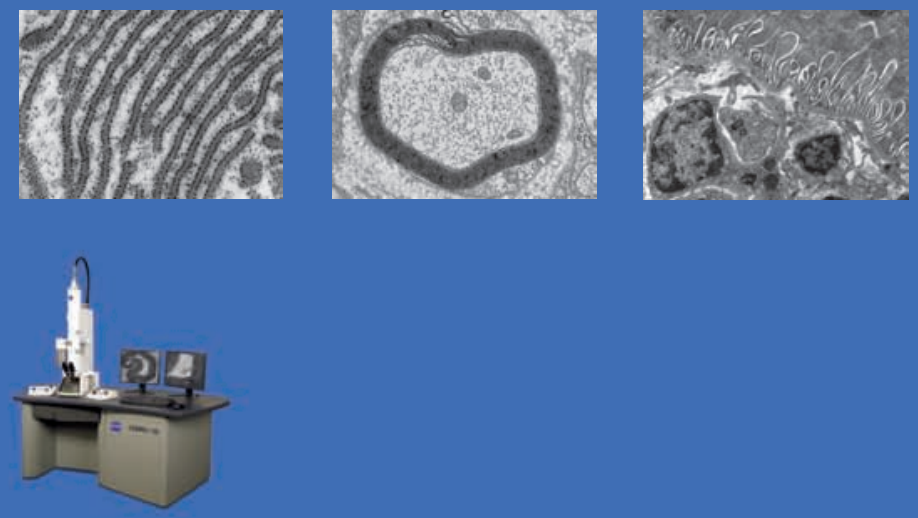

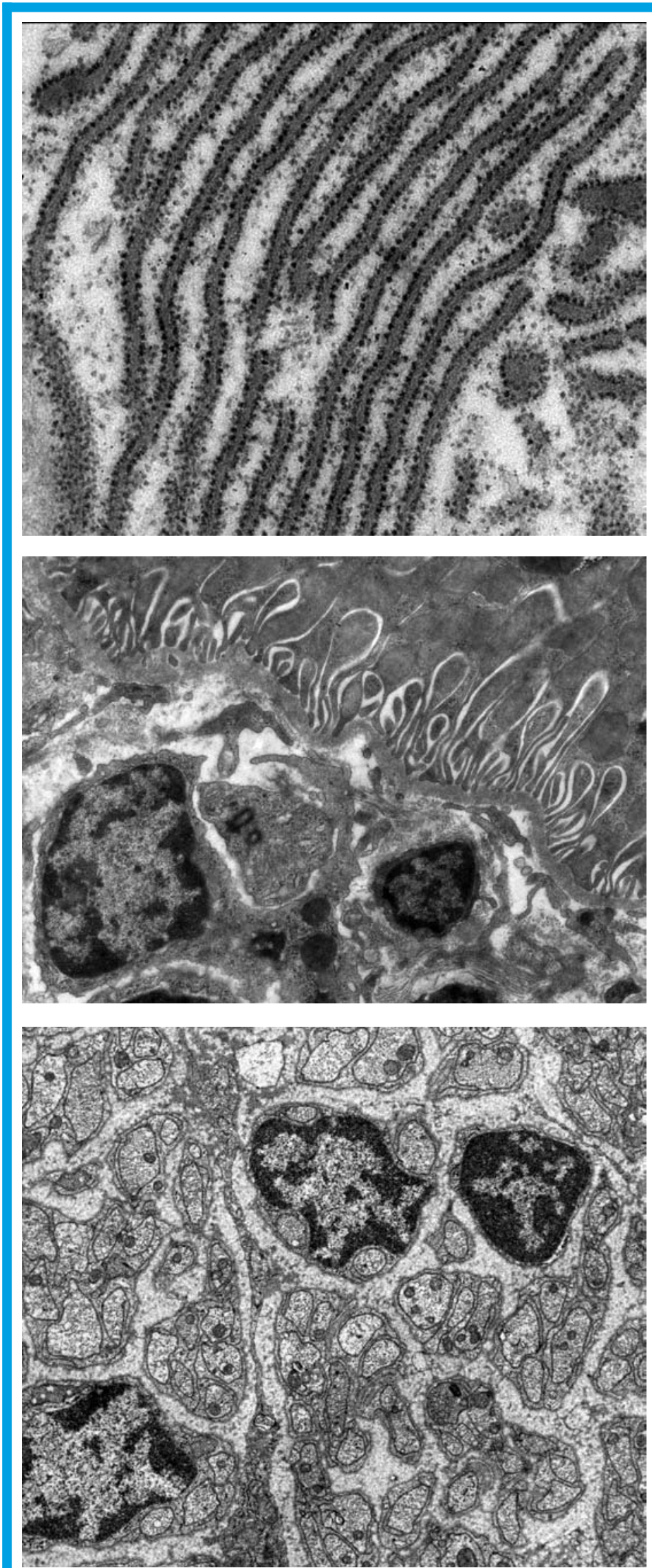

Fig. 4: Imaging of biological samples: (top) pancreatic cell, rough endoplasmic reticulum, (middle) rat kidney, (bottom and top of next column) vagus nerve in CENTRA ${ }^{\oplus} 100$.

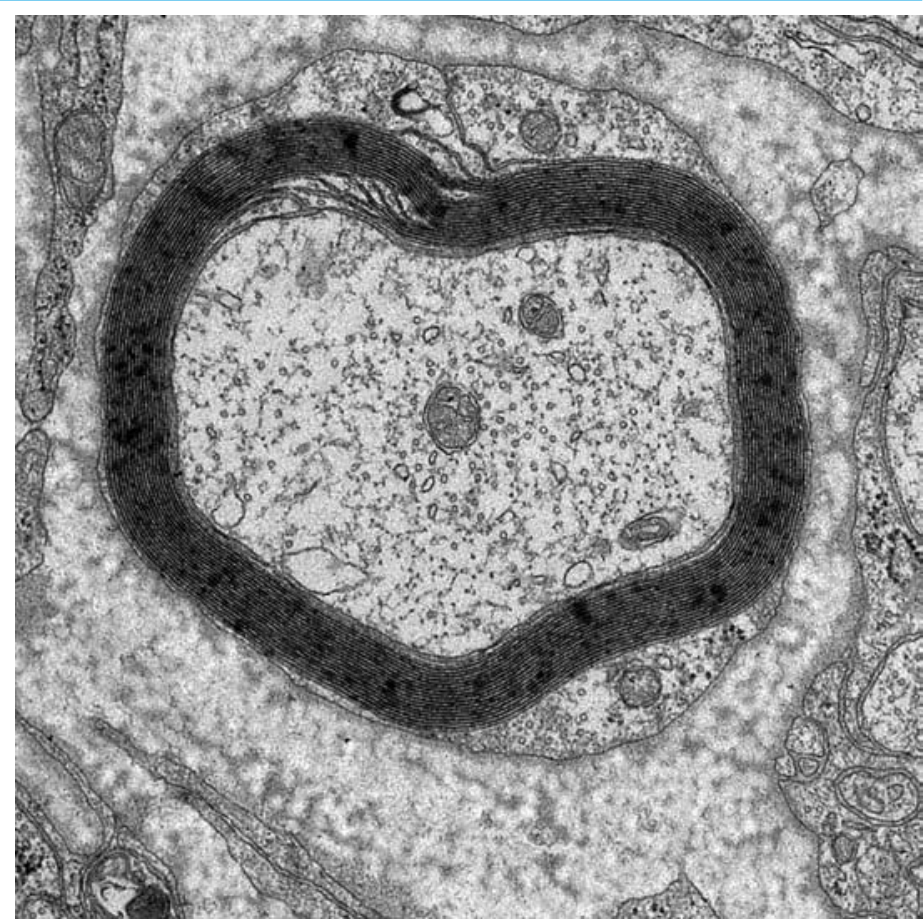

the TEM. (Fig. 2) With this high degree of defined and controlled heat management, much higher current densities can be applied, allowing for the use of minilenses. With the lens size reduced, the entire column can be made smaller. The end result is a compact, economical TEM that fits easily in a standard room. (Fig. 3)

\section{Instrument features}

This new TEM, the CENTRA 100 from Carl Zeiss SMT, provides up to a $100 \mathrm{kV}$ accelerating voltage and up to 0.2 nanometer resolution. Other features of this instrument design include the ability to use two different operating modes-high resolution and high contrast-addressed by means of two different specimen holders. The unique dual-focal-length objective minilens system enables the creation of quality micrographs, even from unstained specimens, which is especially important for the investigation of low-contrast biological samples.

\section{Additional features include:}

- a rotation-free projector system with simplified control of the minilenses,

- a symmetrical objective minilens which reduces aberration coefficients for an enhanced image quality,

- post specimen beam blanking,

- fast, easy air locking for high sample throughput

- an image fine shift system that enables the generation of panoramic images,

- a uniquely simple user interface where two control panels provide total control of the instrument,

- a stable software platform with a Windows-based GUI, and

- cutting edge Slow Scan CCD Cameras.

In addition, learning to operate one of these new TEMs is easy; with only a few hours of training, an operator can be producing quality images efficiently.

Integrated in a small, economical TEM, these features provide an impressive array of imaging capabilities, helping to make the concept of "a TEM for every lab" a reality today.

[1] P.W. Hawkes ed., “Magnetic Electron Lenses”, Springer Verlag, p. 359 (1982) 

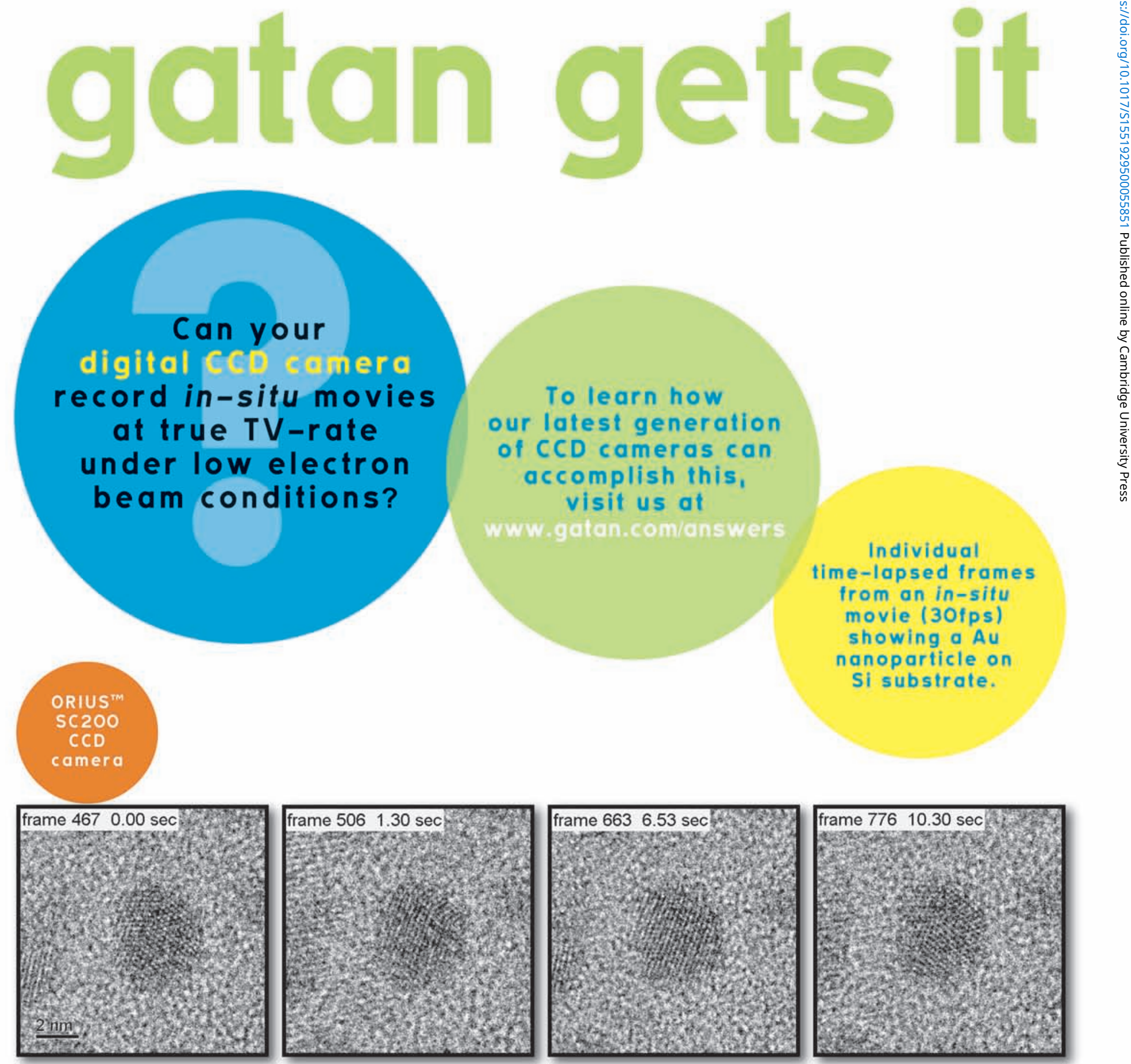

Individual

time-lapsed frames

from an in-sifu

movie ( $30 \mathrm{fps}$ )

showing a $\mathrm{Au}$

nanoparticle on

Si substrate.
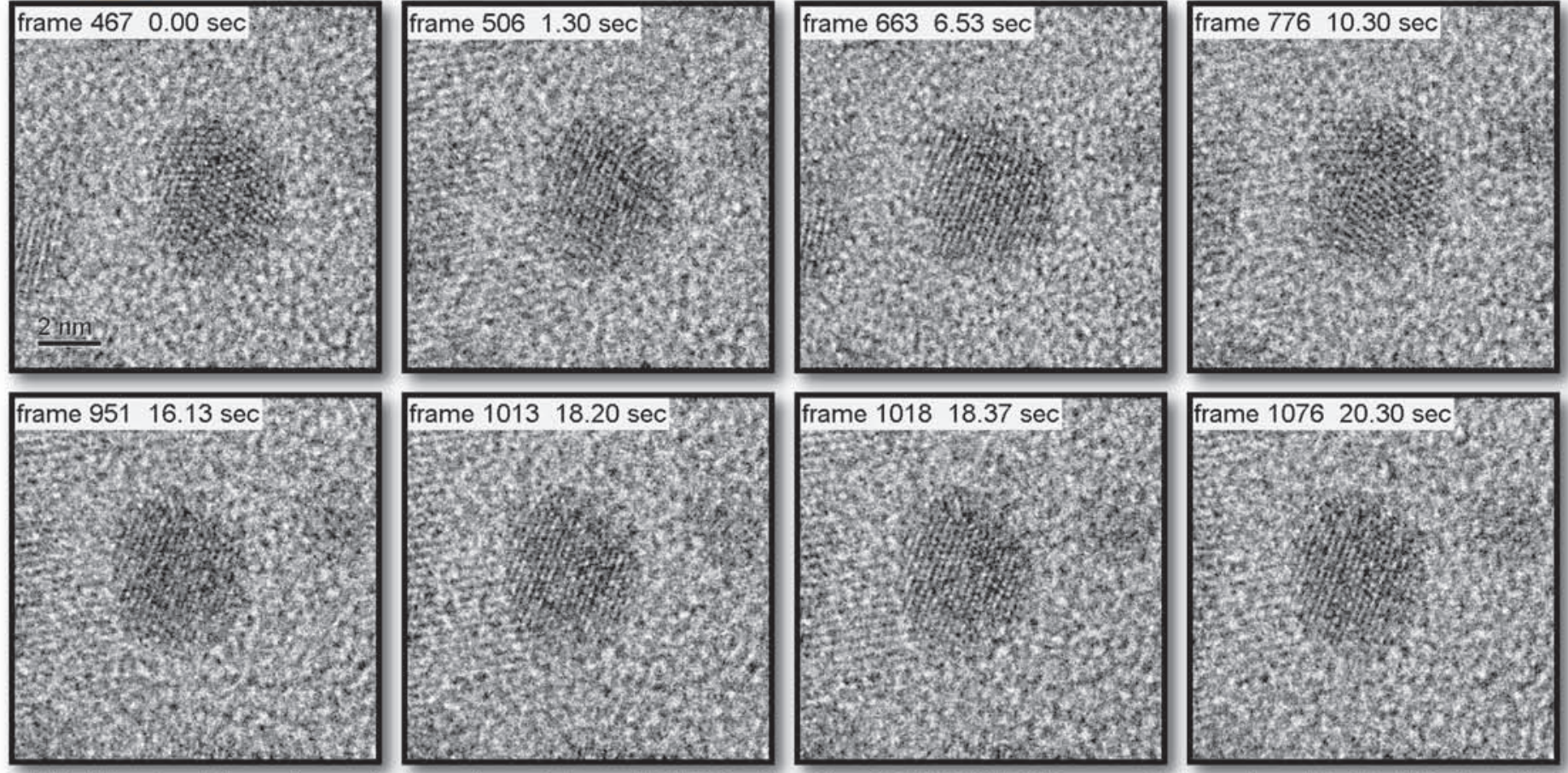

Weiss, Center for Solid State Science (CSSS), Arizona State University.

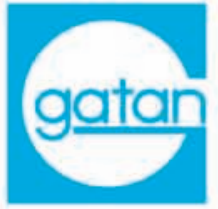

